\title{
Use of Cryptographic Ideas to Interpret Biological Phenomena (and Vice Versa)
}

\author{
Massimo Regoli
}

\section{Introduction}

The RNA-Crypto System (shortly RCS) is a symmetric key algorithm to cipher data. This algorithm, as shown below, has the peculiarity to expand the message to be encrypted hiding the ciphered message itself within a set of garbage and control information.

The idea for this new algorithm starts from the observation of nature. In particular from the observation of RNA behavior and some of its properties.

In particular the RNA sequences has some sections called Introns. Introns, derived from the term "intragenic regions", are non-coding sections of precursor mRNA (pre-mRNA) or other RNAs, that are removed (spliced out of the RNA) before the mature RNA is formed. Once the introns have been spliced out of a pre-mRNA, the resulting mRNA sequence is ready to be translated into a protein. The corresponding parts of a gene are known as introns as well.

The nature and the role of Introns in the pre-mRNA is not clear and it is under ponderous researches by Biologists but, in our case, we will use the presence of Introns, in the RNA-Crypto System output, as a strong method to add only apparently chaotic and non coding information with an unnecessary behavior in the access to the secret key to code the messages.

In the RNA-Crypto System algorithm the introns are sections of the ciphered message with non-coding information as well as in the precursor mRNA. But the term "non-coding" does not necessarily mean "junk data".

In this text a new cryptographic algorithm is described starting from a mathematical point of view.

\section{$1.1 \quad$ Interpretation}

This algorithm can be used to code clear messages (and the main scope of this job regard this application), but it can be used as well as to suggest to biologists to consider redundancy in the pre-mRNA sequences as a mechanism used by nature as a sort of protection against a possible decoding attack from the outside or, from another point of view, they can give to introns an important role in the mechanism for coding the resulting $m R N A$ for example the one to achieve future functionalities or to archive old ones.

\section{Ingredients}

To introduce the RCS algorithm let us start to give some important ingredients.

\section{$2.1 \quad$ Spaces}

We introduce for the first the spaces:

- $\mathcal{K}:=\left\{\kappa_{i} \mid \kappa_{i} \in K\right\}$ as the space of all finite sequences in a given space $K$, of length less or equal to $N_{K}$ where $\infty>N_{K} \in \mathbb{N}$ 
- $\mathcal{M}:=\left\{\sigma_{i} \mid \sigma_{i} \in M\right\}$, as the space of all finite sequences in $M$ of length less or equal to $N_{M}$, where $\infty>N_{M} \in \mathbb{N}$ (could be $M=K$ )

- $S=S_{1} \cup S_{2}$ with $S_{1} \cap S_{2}=\emptyset$ with $|S|<\infty$.

Namely $\mathcal{K}$ is the set of Secret Keys of length $\leq N_{K}, \mathcal{M}$ is the set of Clear Messages of length $\leq N_{M}, K$ and $M$ are finite spaces of symbols (just to fix the ideas should be: $K=M=\{0,1\}$ the standard binary digits). Also $S$ is a finite set of symbols (or functions) and we can consider it as the set of all possible actions that act on the clear message.

At least we introduce the following spaces:

- $\mathcal{O}=\mathcal{A} \cup \mathcal{B}$ where $\mathcal{A}=A^{N_{A}}$ and $\mathcal{B}=\bigcup_{i} \mathcal{B}_{i}$ where $\mathcal{B}_{i}=A^{N_{A}} \times B_{i}^{N_{B_{i}}}$. Also in this case $A$ and $B_{i}$ are finite sets of symbols as above.

From a biological point of view the space $\mathcal{A}$ is the exons space and $\mathcal{B}$ is the introns space.

$\mathcal{O}$ is the base space for Coded Messages (a coded messages will be a finite sequences of elements of $\mathcal{O}$ ). After this preliminary description of necessary spaces, now we can introduce some useful definitions:

Definition 1 Be $\kappa \in \mathcal{K}$. We define for $m \in \mathbb{N}$ and for each $i \in\left\{1, \ldots, N_{K}\right\}$,

$$
\tilde{\kappa}_{i, m}:=\left\{\kappa_{i}, \kappa_{i+1}, \ldots, \kappa_{i \dot{+}(m-1)}\right\}
$$

with $0 \leq m \leq N_{K}$ as a subsequence of $\kappa$

$(m=0$ represent a subsequence of length 0$)$.

Definition 2 Be $\sigma \in \mathcal{M}$. We define for $n \in \mathbb{N}$ and for each $i \in\left\{1, \ldots, N_{M}\right\}$,

$$
\tilde{\sigma}_{i, n}:=\left\{\sigma_{i}, \sigma_{i+1}, \ldots, \sigma_{i \dot{+}(n-1)}\right\}
$$

with $0 \leq n \leq N_{M}$ as a subsequence of $\sigma$.

$(n=0$ represent a subsequence of length 0$)$.

In the above definitions the operator $\dot{\dot{ }}$ is the sum with appropriate module depending on the length of the sequence and, for simplicity, in the following we will use the form $\tilde{\kappa}_{i, m} \equiv \tilde{\kappa}_{i}$ and $\tilde{\sigma}_{i, n} \equiv \tilde{\sigma}_{i}$ moreover holds: $\tilde{\kappa}_{i} \in \mathcal{K}$ and $\tilde{\sigma}_{i} \in \mathcal{M}$

Definition $3 \mathrm{Be} \mathcal{C}=\left\{\Sigma_{i} \mid \Sigma_{i} \in \mathcal{O}\right\}$ the space of all finite sequences in $\mathcal{O}$.

$\mathcal{C}$ is the set of Coded Messages.

\section{$2.2 \quad$ Functions}

To reach our goal we must introduce two kinds of functions:

- Coding functions: these functions are used to:

- transform a portion of the clear message in a portion of the coded message (exons)

- insert some apparently redundant information in the coded message (introns)

- Operational functions: these functions are used to:

- modify the local state of the coding system

- modify the global state of the variables of the system (see below) 
For the first we start with the definition of a family of coding functions $f_{\alpha}: \mathcal{M} \times \mathcal{K} \rightarrow \mathcal{O}$ with $\alpha \in S$ :

$$
f_{\alpha}(\sigma, \kappa)=\Sigma= \begin{cases}\varepsilon & \text { if } \alpha \in S_{1} \\ \iota & \text { if } \alpha \in S_{2}\end{cases}
$$

where $\varepsilon \in \mathcal{A}$ and $\iota \in \mathcal{B}$.

The functions $f_{\alpha}$ must be chosen in such way to guarantee the existence of a function $\bar{f}: \mathcal{O} \times \mathcal{K} \rightarrow \mathcal{M}$ such that

$$
\bar{f}(\Sigma, \kappa)=\bar{f}\left(f_{\alpha}(\sigma, \kappa), \kappa\right)= \begin{cases}\sigma & \text { if } \alpha \in S_{1} \\ \emptyset & \text { if } \alpha \in S_{2}\end{cases}
$$

Now we introduce the definition of some operational functions: be $g_{\alpha}: \mathcal{K} \times \mathcal{O} \rightarrow \mathcal{K}$ as follow:

$$
g_{\alpha}(\kappa, \Sigma)=\bar{g}(\kappa, \Sigma)=\kappa^{*}
$$

the existence of a function $\bar{g}$ that does not depend from $\alpha$ ensures the chaoticity of the system.

For the last, we need, as a member of the set of the operational functions, the trivial characteristic function $\chi_{S_{1}}: S \rightarrow\{0,1\}$

\subsection{Global variables and further definitions}

Now let us introduce some further definitions:

Definition 4 Be $\kappa \in \mathcal{K}$ a Pre Shared Key (PSK) of length $N_{K}$.

In cryptography, a pre-shared key or PSK is a shared secret which was previously shared between the two parties using some secure channel before it needs to be used.

Definition $5 B e\{\alpha\}=\left\{\alpha_{i} \mid \alpha_{i} \in S\right\}$ a random sequence of arbitrary length.

A random sequence is a kind of stochastic process. In short, a random sequence is a sequence of random variables. In computer science it comes from a random number generator, often abbreviated as RNG, that is a computational device designed to generate a sequence of numbers or symbols that lack any pattern, i.e. appear random.

Definition 6 Be $\sigma \in \mathcal{M}$ a message of length $N_{M}$.

In communications science, a message is information which is sent from a source to a receiver.

Definition $7 \Sigma \in \mathcal{C}$ will be the coded message of length that will depend on the system and the variables state.

In cryptography, a coded message is a clear message transformed into an obscured form, preventing those who do not possess special information, or key, required to apply the transform from understanding what is actually transmitted.

\subsection{Biological parallelism}

From the biological point of view the PSK and the random sequence $\{\alpha\}$ represent the whole mechanism of splicing (this is the process by which pre-mRNA is modified to remove certain stretches of non-coding sequences called exactly introns), while the coded message is the pre-mRNA itself and the clear message is the final $m R N A$. 


\section{The algorithm}

\subsection{Coding}

Suppose to have a $\kappa \in \mathcal{K}$ as a Pre Shared Key (PSK) of length $N_{K}$ and a message $\sigma \in \mathcal{M}$ of length $N_{M}$ and that we have fixed two numbers $n, m \in \mathbb{N}$. (For simplicity and without affecting the generality of the system, we can suppose that $N_{M}$ is a multiple of $n$ ).

Using the above definitions for $\alpha_{i}, \tilde{\sigma}_{l_{i}}$ and $\tilde{\kappa}_{j_{i}}$ we can define the following algorithm:

$$
\begin{cases}f_{\alpha_{i}}\left(\tilde{\sigma}_{l_{i}}, \tilde{\kappa}_{j_{i}}\right) & =\Sigma_{i}= \begin{cases}\varepsilon_{i} & \text { if } \alpha_{i} \in S_{1} \\ \iota_{i} & \text { if } \alpha_{i} \in S_{2}\end{cases} \\ l_{i+1} & =l_{i}+\chi_{S_{1}} n \\ \tilde{\kappa}_{j_{i+1}} & =g_{\alpha_{i}}\left(\kappa, \Sigma_{i}\right)\end{cases}
$$

where $\varepsilon_{i} \in \mathcal{A}$ and $\iota_{i} \in \mathcal{B}$.

Definition 8 The sequence $\Sigma=\left\{\Sigma_{1}, \ldots, \Sigma_{N}\right\} \in \mathcal{C}$ is the coded message.

\subsection{Decoding}

For the decoding phase suppose to have $\kappa \in \mathcal{K}$ as a Pre Shared Key (PSK) of length $N_{K}$ and a coded message $\Sigma \in \mathcal{C}$ of arbitrary length, and suppose that we have fixed two numbers $n, m \in \mathbb{N}$.

Using the above definitions for $\tilde{\sigma}_{l_{i}}$ and $\tilde{\kappa}_{j_{i}}$ we can define the following algorithm:

$$
\begin{cases} \begin{cases}\bar{f}\left(\Sigma_{i}, \tilde{\kappa}_{j_{i}}\right) & =\tilde{\sigma}_{i} \\
\tilde{\kappa}_{j_{i+1}} & =\bar{g}\left(\kappa, \Sigma_{i}\right)\end{cases} & \text { if } \Sigma_{i} \in \mathcal{A} \\
\left\{\begin{array}{ll}
\bar{f}\left(\Sigma_{i}, \tilde{\kappa}_{j_{i}}\right) & =\emptyset \\
\tilde{\kappa}_{j_{i+1}} & =\bar{g}\left(\kappa, \Sigma_{i}\right)
\end{array} \quad \text { if } \Sigma_{i} \in \mathcal{B}_{*} \subset \mathcal{B}\right.\end{cases}
$$

Note that, thanks to the definition of $\bar{g}$, in the decoding phase is not necessary to know the random $\left\{\alpha_{i}\right\}$ sequence.

\subsection{Preliminary observations}

The coded message depends on:

- The clear message;

- The secret Key (PSK);

- The random sequence $\{\alpha\}$;

and it important to understand that the algorithm during the coding phase using the same message and the same PSK can supply different coded messages simply using different $\{\alpha\}$.

It is also important to underline the fact that it is no necessary, during the decoding phase, to know the sequence $\{\alpha\}$ used in the coding phase. This because the information about the $\{\alpha\}$ sequence are intrinsic in the couple $\left(\Sigma_{i}, \tilde{\kappa}_{j_{i}}\right)$.

In this circumstance we want to underline the possible role of introns to carry important information for the decoding phase, for example the function $\bar{g}(\kappa, \Sigma)$ can use the information present in $\Sigma \in \mathcal{B}$ to produces a change in the PSK.

Moreover, during the coding phase there is an expansion of the original messages into the coded message, and this expansion comes from several factors, for example it comes from the dimensions of the $\mathcal{B}_{i}$ and from the number of elements of $S_{2}$ in the $\{\alpha\}$ sequence. 


\section{Early implementations}

\subsection{The environment}

Now let us introduce the first realization of the above algorithm. Step by step we will substitute each ingredient of the algorithm, with its representative object in the implementation.

Starting from the definition of $K=M=A=B_{1}=\{0,1\}$, with $N_{K}, N_{M} \in \mathbb{N}, N_{A}=3, N_{B_{1}}=2$ and $S=S_{1} \cup S_{2}$ with $S_{1}=\{a, b\}$ and $S_{2}=\{c, d\} . \mathcal{O}=\{0,1\}^{N_{A}} \times\left(\{0,1\}^{N_{A}} \times B_{1}^{N_{B_{1}}}\right)$. Finally $n=1$ and $m=3$.

\subsection{Functions and implementation}

To introduce the functions $f_{\alpha}$ the table $1 T: \mathcal{A} \times K^{n} \rightarrow S$ will be useful using the following rules:

1) if $\alpha \in S_{1}$

- be $c=\tilde{\kappa}_{j}$

- if $\tilde{\sigma}_{i}=\sigma_{i}=0$ then localize a binary number $000 \leq r \leq 111$ such that $T_{r, c}=a\left(\right.$ or $\left.T_{r, c}=b\right)$

- if $\tilde{\sigma}_{i}=\sigma_{i}=1$ then localize a binary number $000 \leq r \leq 111$ such that $T_{r, c}=b\left(\right.$ or $\left.T_{r, c}=a\right)$

- now let $\Sigma_{i}=\left(\Sigma_{i, 1}, \Sigma_{i, 2}, \Sigma_{i, 3}\right)=\left(r_{1}, r_{2}, r_{3}\right)$ (i.e. an exon $\left.\Sigma_{i} \in \mathcal{A}\right)$.

2) if $\alpha \in S_{2}$

- be $c=\tilde{\kappa}_{j}$

- localize a binary number $000 \leq r \leq 111$ such that $T_{r, c}=\alpha$

- now let $\Sigma_{i}=\left(\left(\Sigma_{i, 1}, \Sigma_{i, 2}, \Sigma_{i, 3}\right),\left(\Sigma_{i, 4}, \Sigma_{i, 5}\right)\right)=\left(\left(r_{1}, r_{2}, r_{3}\right),\left(\Sigma_{i, 4}, \Sigma_{i, 5}\right)\right)$, (i.e. an intron $\left.\Sigma_{i} \in \mathcal{B}\right)$

- the other 2 components of the vector (i.e. $\left.\Sigma_{i, 4}, \Sigma_{i, 5}\right)$ will be given using a random choice in the binary range $\{00, \ldots, 11\}$

\begin{tabular}{|c|c|c|c|c|c|c|c|c|c|}
\hline \multicolumn{2}{|c|}{} & \multicolumn{9}{|c|}{$\tilde{\kappa}_{j}$} \\
\cline { 2 - 12 } & 000 & 001 & 000 & 011 & 100 & 101 & 110 & 111 \\
\hline \hline \multirow{5}{*}{$\left(\Sigma_{i, 1}, \Sigma_{i, 2}, \Sigma_{i, 3}\right)$} & 000 & $\mathrm{a}$ & $\mathrm{b}$ & $\mathrm{c}$ & $\mathrm{d}$ & $\mathrm{a}$ & $\mathrm{b}$ & $\mathrm{c}$ & $\mathrm{d}$ \\
\cline { 2 - 13 } & 001 & $\mathrm{~b}$ & $\mathrm{c}$ & $\mathrm{d}$ & $\mathrm{a}$ & $\mathrm{b}$ & $\mathrm{c}$ & $\mathrm{d}$ & $\mathrm{a}$ \\
\cline { 2 - 12 } & 010 & $\mathrm{c}$ & $\mathrm{d}$ & $\mathrm{a}$ & $\mathrm{b}$ & $\mathrm{c}$ & $\mathrm{d}$ & $\mathrm{a}$ & $\mathrm{b}$ \\
\cline { 2 - 12 } & 011 & $\mathrm{~d}$ & $\mathrm{a}$ & $\mathrm{b}$ & $\mathrm{c}$ & $\mathrm{d}$ & $\mathrm{a}$ & $\mathrm{b}$ & $\mathrm{c}$ \\
\cline { 2 - 12 } & 100 & $\mathrm{a}$ & $\mathrm{b}$ & $\mathrm{c}$ & $\mathrm{d}$ & $\mathrm{a}$ & $\mathrm{b}$ & $\mathrm{c}$ & $\mathrm{d}$ \\
\cline { 2 - 11 } & 101 & $\mathrm{~b}$ & $\mathrm{c}$ & $\mathrm{d}$ & $\mathrm{a}$ & $\mathrm{b}$ & $\mathrm{c}$ & $\mathrm{d}$ & $\mathrm{a}$ \\
\cline { 2 - 11 } & 110 & $\mathrm{c}$ & $\mathrm{d}$ & $\mathrm{a}$ & $\mathrm{b}$ & $\mathrm{c}$ & $\mathrm{d}$ & $\mathrm{a}$ & $\mathrm{b}$ \\
\cline { 2 - 11 } & 111 & $\mathrm{~d}$ & $\mathrm{a}$ & $\mathrm{b}$ & $\mathrm{c}$ & $\mathrm{d}$ & $\mathrm{a}$ & $\mathrm{b}$ & $\mathrm{c}$ \\
\hline
\end{tabular}

Table 1: The seek table $T_{r, c}$

Now for the $\bar{g}$ function we will use the following rules:

- obtain $r, c$ as above

- if $T_{r, c} \in\{a, b\}$ then $\bar{g}$ will return $\tilde{\kappa}_{j_{i}+m}$

- if $T_{r, c}=c$ then $\bar{g}$ will return $\tilde{\kappa}_{j_{i}+\left(\Sigma_{i, 4} \Sigma_{i, 5}\right)_{10}}$

- if $T_{r, c}=d$ then $\bar{g}$ will return $\tilde{\kappa}_{j_{i}-\left(\Sigma_{i, 4} \Sigma_{i, 5}\right)_{10}}$ 
where the subscript 10 means the decimal representation of the binary number in parenthesis.

The last function we must define is $\bar{f}: \mathcal{O} \times \mathcal{K} \rightarrow \mathcal{M}$. Remember that conditions in equation (2) must hold.

The function $\bar{f}\left(\Sigma_{i}, \tilde{\kappa}_{j}\right)$ could be able to understand if $\Sigma_{i} \in \mathcal{A}$ or $\Sigma_{i} \in \mathcal{B}$. Starting from the fact that $\Sigma$ (i.e. the entire coded message) is a sequence of 0 and 1 , we can extract the single element in terms of bits (i.e. $\left.\tilde{\Sigma}_{l_{i}}, \tilde{\Sigma}_{l_{i}+1}, \ldots\right)$. Following this strategy we can define:

$$
\bar{f}\left(\Sigma_{i}, \tilde{\kappa}_{j_{i}}\right):=\bar{f}\left(\left(\tilde{\Sigma}_{l_{i}}, \tilde{\Sigma}_{l_{i}+1}, \ldots\right), \tilde{\kappa}_{j_{i}}\right)
$$

the new function act in this way:

- be $r=\left(\tilde{\Sigma}_{l_{i}}, \tilde{\Sigma}_{l_{i}+1}, \tilde{\Sigma}_{l_{i}+2}\right)$

- be $c=\tilde{\kappa}_{j_{i}}$

- if $T_{r, c}=a$ then $\bar{f}$ returns $\sigma_{i}=\{0\}$ (or $\left.\sigma_{i}=\{1\}\right)$

- if $T_{r, c}=b$ then $\bar{f}$ returns $\sigma_{i}=\{1\}$ (or $\left.\sigma_{i}=\{0\}\right)$

- else $\bar{f}$ return $\sigma_{i}=\emptyset$

The function $\bar{g}$ acts as above but using $\left(\tilde{\Sigma}_{l_{i}}, \tilde{\Sigma}_{l_{i}+1}, \tilde{\Sigma}_{l_{i}+2}\right)$ if $T_{r, c}=\{a, b\}$ or $\left(\tilde{\Sigma}_{l_{i}}, \tilde{\Sigma}_{l_{i}+1}, \tilde{\Sigma}_{l_{i}+2}, \tilde{\Sigma}_{l_{i}+3}, \tilde{\Sigma}_{l_{i}+4}\right)$ if $T_{r, c}=\{c, d\}$.

\subsection{Observation}

In this simple implementation of the RNA algorithm, redundancy is given by the following considerations:

- for each processed bit in the clear message 3 bits will be inserted in the coded one

- each intron will insert in the coded message 5 bits

- we use an uniform distribution for the generation of the sequence $\left\{\alpha_{i}\right\}$

- then we can suppose that the length of the sequence $\left\{\alpha_{i}\right\}$ is $\left\|\left\{\alpha_{i}\right\}\right\|=\left\|\left\{\alpha_{i} \mid \alpha_{i} \in S_{1}\right\}\right\|+\left\|\left\{\alpha_{i} \mid \alpha_{i} \in S_{2}\right\}\right\|$

- of course, in this implementation, must be $\left\|\left\{\alpha_{i} \mid \alpha_{i} \in S_{1}\right\}\right\|=N_{M}$

Then, if the original message has a length of $N_{M}$ bits, the length of coded one is $l_{c} \approx 3 N_{M}+5 N_{M}=8 N_{M}$.

But it is important to underline that this implementation uses a redundant table $T_{r, c}$, in fact for each $c$ there are two $r$ that satisfy the condition $T_{r, c}=\alpha$.

But of course if we want to reduce the expansion of the message we can replace it with the one in table 2 (in this version must be $N_{A}=2$ and $m=2$ ).

\begin{tabular}{|c|c|c|c|c|c|}
\hline & \multicolumn{4}{|c|}{$\tilde{\kappa}_{j}$} \\
\hline & & 00 & 01 & 10 & 11 \\
\hline \multirow{4}{*}{$\left(\Sigma_{i, 1}, \Sigma_{i, 2}\right)$} & 00 & $\mathrm{a}$ & $\mathrm{b}$ & $\mathrm{c}$ & $\mathrm{d}$ \\
\hline & 01 & $b$ & $\mathrm{c}$ & $\mathrm{d}$ & $\mathrm{a}$ \\
\hline & 10 & $\mathrm{c}$ & $\mathrm{d}$ & $\mathrm{a}$ & $\mathrm{b}$ \\
\hline & 11 & $\mathrm{~d}$ & $\mathrm{a}$ & $b$ & $\mathrm{c}$ \\
\hline
\end{tabular}

Table 2: An alternative seek table $T_{r, c}$

in this case the message explosion will be $l_{c} \approx 2 N_{M}+4 N_{M}=6 N_{M}$. 
Another solution in order to diminish the explosion of the coded message is that to use an asymmetric probability function for the generation of the $\left\{\alpha_{i}\right\}$ for example:

\begin{tabular}{|c|c|}
\hline$\alpha$ & $\mathrm{p}$ \\
\hline \hline $\mathrm{a}$ & $3 / 8$ \\
\hline $\mathrm{b}$ & $3 / 8$ \\
\hline $\mathrm{c}$ & $1 / 8$ \\
\hline $\mathrm{d}$ & $1 / 8$ \\
\hline
\end{tabular}

Table 3:

Using these last two optimizations the approximate length of the coded message will be reduced to $l_{c} \approx 3 N_{M}$.

\subsection{A biological implementation}

Now, as an example, we want to create an implementation of our model nearer the biology. For this let us introduce again the ingredients for the new model:

Starting from the definition of $K=\mathbb{N}, M=A=\{a, b, c, d\}$, with $N_{K}, N_{M} \in \mathbb{N}$, and $S=S_{1} \cup S_{2}$ with $S_{1}=\{c\}$ and $S_{2}=\{n c\} . \mathcal{O}=\left\{A^{N_{A}} \backslash(a, a, a)\right\} \cup\left(\{a, a, a\} \times\left(\bigcup_{i} A^{N_{B_{i}}}\right)\right), N_{A}=n=3, N_{B_{i}} \in \mathbb{N}$ and $m=1$.

The function $f$ will be:

$$
\Sigma_{i^{*}}=f_{\alpha}\left(\tilde{\sigma}_{i}, \tilde{\kappa}_{j_{i}}\right)= \begin{cases}\tilde{\sigma}_{i} & \text { if } \alpha \in S_{1} \\ (a, a, a, B) \text { with } B \in A^{\tilde{\kappa}_{j_{i}}} & \text { if } \alpha \in S_{2}\end{cases}
$$

in this case the element $(a, a, a) \in A^{3}$ is the codon to localize the beginning of the introns in the sequence (it will never appear in the clear code) and the function $\bar{f}$ will be:

$$
\sigma_{i^{*}}=\bar{f}\left(\Sigma_{i}, \tilde{\kappa}_{j_{i}}\right)= \begin{cases}\left(\Sigma_{i, 1}, \Sigma_{i, 2}, \Sigma_{i, 3}\right) & \text { if }\left(\Sigma_{i, 1}, \Sigma_{i, 2}, \Sigma_{i, 3}\right) \neq(a, a, a) \\ \emptyset & \text { otherwise }\end{cases}
$$

In both cases holds $\tilde{\kappa}_{j_{i+1}}=\tilde{\kappa}_{j_{i}+1}$.

\section{The role of the Coding Functions}

In section 2.2 we introduce the definition of Coding functions.

These functions play an important role in the complexity and in the lengths of the cipher text.

It is also very important to understand that the number of functions involved in the process of encryption can be huge. In fact, besides the canonical functions introduced in section 4.1 we can add functions that have the role of:

- Change the public / secret keys

- For example in the cipher text we can insert a sequence of bits of arbitrary length that will replace the public (private) key to decode the rest of the message.

- Insert a long sequences of random bits

- As before but the inserted bits are ignored.

- Move forward or backward the key pointer position 
- The presence of a disruptive effect on the sequencing access to the secret key can only add more noise in the attacks

- Reset global parameters like message pointer position, key pointer position, secret and public data

- See before

is easy to see that the number of Coding functions that can be inserted in the encoding mechanism is great and may become part of the shared secret information.

In the same way it is obvious that some Coding functions can greatly increase the size of the cipher text as it is also obvious that the inclusion of random bits within the text could create an increase in the randomness of the cipher text.

\section{Statistical Analysis}

\subsection{Cryptanalysis}

Cryptanalysis (from the greek krypts, "hidden" and analein, "break") is the study of methods for obtaining the meaning of encrypted information without having access to secret information which is usually required to perform the operation. Typically it comes to finding a secret key.

Cryptanalysis is the "counterpart" of cryptography, namely the study of techniques for concealing a message, and together form the cryptology, the science of writing hidden.

Cryptanalysis refers also to any attempt to circumvent the security of other cryptographic algorithms and cryptographic protocols.

Although the methods of cryptanalysis usually excludes attacks that are directed to the inherent weaknesses of the method to violate, such as bribery, physical coercion, theft, social engineering, such attacks are often the most productive of cryptanalysis traditional, they are still an important component.

The first cryptanalytic tool used to analyze the RNA-Crypto System is based on the Statistical analysis. For this purpose we used a famous battery of tests called Diehard (or Dieharder in the new version).

- The Diehard tests is a battery of statistical tests for measuring the quality of a set of random numbers.

- It is cited by NIST as one of the best statistical suite for testing randomness

- It was developed by George Marsaglia over several years and first published in 1995 and then maintained and improved by Robert Brown at the Duke University.

- We focused our attention to the following tests:

1. Birthday spacings

2. Overlapping permutations

3. Ranks of matrices

4. Monkey tests

5. Count the $1 \mathrm{~s}$

6. Parking lot test

7. Minimum distance test

8. Random spheres test

9. The squeeze test

10. Overlapping sums test

11. Runs test

12. The craps test

all these tests are well described in the software package and in literature. 


\begin{tabular}{|l|l|}
\hline base & binary \\
\hline $\mathrm{a}$ & 00 \\
$\mathrm{c}$ & 01 \\
$\mathrm{~g}$ & 10 \\
$\mathrm{t} \mid \mathrm{u}$ & 11 \\
\hline
\end{tabular}

Table 4: Translation table

\subsection{Our Idea}

In nature a nucleotides ribbon is a sequence of (almost always) 4 symbols (a, c, g, t $\mid \mathrm{u})$.

In computer science a 'string' is a sequence of 2 symbols $(0,1)$.

Now, suppose to translate the 4 symbols as in table 4: then we have a correspondence between bits and nucleotides.

With this assumption the Diehard tests can be used to assess the randomness of this binary conversion of a sequence of nucleotides as well as the sequence of an encrypted message.

Our idea is based on the following questions:

1. Have RNA ribbons a good random behavior according to DieHard(er) tests?

2. Are RNA-Crypto System sequences a good random behavior according to DieHard(er) tests?

The answers can be given by looking at test results.

\subsection{BIO results}

\subsubsection{The Experiment}

We used only some very long sequences of nucleotides from on line standard free databases, this because the tests run well on more than $12 \mathrm{M}$ bytes of data.

Accordingly with some simple calculations, holds the formula $1 \mathrm{M}$ bytes $=\frac{1}{4} \mathrm{M}$ bases, then we need sequences of at least $48 \mathrm{M}$ bases, so our attention was focused also on the human genome which can gave very long sequences.

\subsubsection{The protocol}

1. Get a sequence from the database (longer than $48 \mathrm{M}$ bases)

2. Translate it in binary mode

3. Run the DH test on it

\subsubsection{The Data}

For the biological sequences we uses the following:

- Caenorhabditis elegans chromosomes I-V, complete sequences.

- Wallaby, whole genome.

- Human chromosome 14 complete sequence

- Drosophila melanogaster some chromosomes, complete sequences

all encoded use table 4. 


\subsubsection{The Results}

In table 5 we can see the results of our experiment using the Biological data. It is obvious that Bio-sequences are not random at all.

The reason for this fact could be found, of course, in:

- Some parts of a pre-mRNA sequence could be highly repetitive (Satellite, Minisatellite, ...)

- Some parts of a pre-mRNA sequence could be made up a very long sequence of the same nucleotide (Polyadenylation tail, ...)

\begin{tabular}{|r|l|l|}
\hline $\mathrm{n}$ & Test name & Status \\
\hline 1 & Birthday Spacings & FAIL \\
2 & Overlapping Permutations & FAIL \\
3 & Ranks of 31x31 and 32x32 matrices & FAIL \\
4 & Ranks of 6x8 Matrices & FAIL \\
5 & Monkey Tests on 20-bit Words & FAIL \\
6 & Monkey Tests OPSO,OQSO,DNA & FAIL \\
7 & Count the 1's in a Stream of Bytes & FAIL \\
8 & Count the 1's in Specific Bytes & FAIL \\
9 & Parking Lot Test & FAIL \\
10 & Minimum Distance Test & FAIL \\
11 & Random Spheres Test & FAIL \\
12 & The Squeeze Test & FAIL \\
13 & Overlapping Sums Test & FAIL \\
14 & Runs Test & FAIL \\
15 & The Craps Test & FAIL \\
\hline
\end{tabular}

Table 5: Bio results

\subsection{RNA-Crypto System results}

\subsubsection{The Experiment}

In the RNA-Crypto System experiment we long sequences of binary data encrypted with our protocol (approximately 100 MBytes of data for each experiment).

\subsubsection{The Protocol}

The protocol used to estimate the randomness of RNA-Crypto System is:

1. Run the DNACrypto program on a message of opportune length

2. Save the encrypted message

3. Run the DH test considering it as a random sequence.

\subsubsection{The Data}

For the cryptographic sequences we uses:

- A 10 M bytes file filled with ASCII char 'A'

- A 10 M bytes file filled with random binary numbers 
- One of the above biological sequence

encoded with the Algorithm

\subsubsection{The Results}

In table 6 we can see the results of our experiment using the RNA-Crypto System data. It is obvious that RNA-Crypto System sequences are random following the DH tests.

\begin{tabular}{|r|l|l|}
\hline $\mathrm{n}$ & Test name & Passed \\
\hline 1 & Birthday Spacings & PASS \\
2 & Overlapping Permutations & PASS \\
3 & Ranks of 31x31 and 32x32 matrices & PASS \\
4 & Ranks of 6x8 Matrices & PASS \\
5 & Monkey Tests on 20-bit Words & PASS \\
6 & Monkey Tests OPSO,OQSO,DNA & PASS \\
7 & Count the 1's in a Stream of Bytes & PASS \\
8 & Count the 1's in Specific Bytes & PASS \\
9 & Parking Lot Test & PASS \\
10 & Minimum Distance Test & PASS \\
11 & Random Spheres Test & PASS \\
12 & The Squeeze Test & PASS \\
13 & Overlapping Sums Test & PASS \\
14 & Runs Test & PASS \\
15 & The Craps Test & PASS \\
\hline
\end{tabular}

Table 6: Crypto results

\subsection{First Results - Differences}

As expected the results correspond to our ideas on the sequences of nucleotides and maybe also to the ones about the Crypto System. Of course natural phenomena is not really random like a cryptographic system Some obvious questions are:

- Why pre-mRNA sequences do not pass the statistical tests?

- Of course they are not random (life is not random)

- But some of the motivations, from the statistical point of view, may be the follows

- Some parts of a pre-mRNA sequence could be highly repetitive (Satellite, Minisatellite, ...)

- Some parts of a pre-mRNA sequence could be made up a very long sequence of the same nucleotide (Polyadenylation tail, ...)

- Is is possible to manipulate the RNA-Crypto System protocol to obtain the same results?

\subsection{Changes: Informatics emulates biology}

\subsubsection{Modify Cryptographic protocol - Phase I}

Due to its random nature the cryptographic model has not repeated sequences inside then we introduce a new coding function $\rho$ (the replicator function) that will add these repeated sequences artificially inside the code

These code can be considered: 
- active (they act in some way with the system)

- passive (just junk!!!)

\subsubsection{Strategies}

The replicator function

Definition $\operatorname{Be} \rho: \mathcal{M} \times \mathcal{K} \times \mathcal{O} \rightarrow \mathcal{O}$ a junk function:

$$
\rho(\sigma, \kappa, o)=\Sigma=\iota
$$

where $\mathcal{B} \ni \iota=o_{\sigma, \kappa}$ and $o_{\sigma, \kappa}$ is a subsequence of $o$.

If $o$ is a part of the encoded message, this mechanism implements the repeated sequences phenomenon.

\subsubsection{Alter Cryptographic protocol - Phase II}

Due to its random nature the cryptographic model has not significant all-equal subsequences then we introduce a new coding function $\tau$ (stutter function) that will add these mono-symbols subsequences artificially.

Also in this case this sub-sequences can be created to be:

- active (they act in some way with the system)

- passive (just junk!!!)

\subsubsection{Phase II - Strategies}

The stutter function

Definition $\operatorname{Be} \tau: \mathcal{M} \times \mathcal{K} \rightarrow \mathcal{O}$ a junk function:

$$
\tau(\sigma, \kappa)=\Sigma=\iota
$$

where $\mathcal{B} \ni \iota=\tilde{\iota}^{n_{\sigma, \kappa}}$ and $\tilde{\iota} \in A \cup B_{i}, n_{\sigma, \kappa}$ is an integer.

This mechanism implements the polyadenylation tail.

\subsection{Results}

New results come from statistical analysis on the new cryptographic data.

Note: the result in table 7 holds for all the data in the cryptographic set

\subsection{New Results - Differences}

In these days we are working hard to prove that the security of the cryptographic system is not affected by the new entrants, nowadays we did not find any attack that can exploit these features, so we are highly confident that the new system has the same level of security of the standard one.

- In fact, even if the spy could isolate the Introns, the rest of the message would be exactly equal to the previous version

- Then the safety remains the same (or better)

Moreover adding redundancy we obtained, as a side effect, some properties:

- A certain robustness to error in transmission 


\begin{tabular}{|r|l|l|}
\hline $\mathrm{n}$ & Test name & Status \\
\hline 1 & Birthday Spacings & FAIL \\
2 & Overlapping Permutations & FAIL \\
3 & Ranks of 31x31 and 32x32 matrices & FAIL \\
4 & Ranks of 6x8 Matrices & FAIL \\
5 & Monkey Tests on 20-bit Words & FAIL \\
6 & Monkey Tests OPSO,OQSO,DNA & FAIL \\
7 & Count the 1's in a Stream of Bytes & FAIL \\
8 & Count the 1's in Specific Bytes & FAIL \\
9 & Parking Lot Test & FAIL \\
10 & Minimum Distance Test & FAIL \\
11 & Random Spheres Test & FAIL \\
12 & The Squeeze Test & FAIL \\
13 & Overlapping Sums Test & FAIL \\
14 & Runs Test & FAIL \\
15 & The Craps Test & FAIL \\
\hline
\end{tabular}

Table 7: New crypto results

- Indeed, in a very redundant system, the probability that an error in a bit prevents the decoding is very low

- This also suggests a particular interpretation of redundancy in RNA (protection against excessive mutations as an example)

- Furthermore, the spy, during his attack, does not know whether the piece of code that is trying to attack be an Exon or an Intron

- This also suggests another particular interpretation of redundancy in RNA (protection against pathogens ?)

\section{Conclusion}

In conclusion this job will allow us to take some considerations.

For the first, adding to a good random sequence some artificial noise, we transformed it in a non random sequence but this transformation does not affect the cryptographic security.

Then this means that tests of randomness, while giving a good estimate of safety, are not always infallible and more tests are needed to determine the goodness of an encryption system.

Finally, the similarity between biology and computer science (cryptography) must be investigated further in order to give further interpretation to the biological mechanisms also today still partially obscure.

\section{References}

[1] Lewin, B., "Gene VI" Oxford University Press, 1997.

[2] Menezes, A., van Oorschot, P. and Vanstone, S., "Handbook of Applied Cryptography", CRC Press, 1996

[3] Regoli, M., "Bio-Cryptography: A Possible Coding Role for RNA Redundancy", Foundations of Probability and Physics-5 (AIP Conference Proceedings), Accardi EDT, 2008 